\title{
Progrès récents en métrologie diphasique
}

\section{Recent progress in two-phase metrology}

\author{
François Resch \\ Chargé de Recherche au C.N.R.S. \\ Institut de Mécanique Statistique de la Turbulence \\ de Marseille*
}

\section{Introduction}

Une revue des progrès récents en métrologie diphasique est une entreprise sans doute trop ambitieuse si elle ne comporte dès le départ un certain nombre de restrictions. Comme il est évident que cette revue ne peut être exhaustive, il semble en effet préférable de concentrer notre attention sur un nombre restreint de techniques récemment mises au point ou en état de développement avancé plutôt que d'énumérer une liste plus ou moins commentée d'un plus grand nombre de techniques dont certaines sont moins employées que d'autres.

Par ailleurs les travaux [1] du séminaire "Métrologie des écoulements diphasiques" de la S.H.F. (1) ont permis de dégager certaines méthodologies capables de retenir notre intérêt dans le présent cadre.

Nous nous bornerons donc à décrire brièvement quelques méthodes et techniques développées seulement en France au cours de cette dernière décade. Nous avons ainsi retenu :

- la mesure des aires interfaciales et des coefficients de transfert de matière par une méthode chimique ;

- la mesure du frottement pariétal en écoulement diphasique par une méthode électrochimique ;

- la détermination de la fonction de phase en écoulement diphasique finement dispersé par une méthode optique de réfraction totale;

- la détermination de la granulométrie de bulles d'air ou de gouttes d'eau de faible diamètre (quelques

$\left.{ }^{*}\right)$ Institut de Mécanique Statistique de la Turbulence Laboratoire associé au C.N.R.S. n⿳ $130 ; 12$, avenue Général Leclerc, 13003 Marseille.

(1) Séminaire "Métrologie des écoulements diphasiques" du groupe de travail "Ecoulements polyphasiques" de la S.H.F. dirigé par Monsieur H. Mondin. Ce séminaire est animé par F. Resch. microns et plus) par une méthode optique de diffusion ;

-- le traitement numérique du signal de sortie d'un anémomètre à film chaud dans le cas d'un écoulement à poches.

Les trois premiers ont été développés dans des laboratoires français dont nous citerons le nom, ainsi que celui des auteurs qui ont contribué à ces travaux. C'est avec leur autorisation que nous présentons leurs résultats; nous les remercions de leur coopération. Les deux derniers sujets ont été étudiés par l'auteur.

Méthode chimique : mesure des aires interfaciales et des coefficients de transfert de matière

Cette méthode a été développée et mise en application à l'Ecole Nationale Supérieure des Industries Chimiques de l'Institut National Polytechnique de Lorraine (à Nancy) par Messieurs J.C. Charpentier, A. Laurent et C. Prost (Voir référence [2]).

C'est une méthode globale (et non locale) de détermination de l'aire interfaciale, $a$, et des coefficients de transfert de matière, $k_{L} a$ et $k_{G} a$ ( $k_{L}$ et $k_{G}$ étant les conductances partielles de transfert dans les phases liquides et gazeuses). Ces deux paramètres ont une importance fondamentale en génie chimique car c'est d'eux que dépendent la caractérisation et l'efficacité des contacteurs gaz-liquides dont l'emploi est bien connu.

Le principe de la méthode est le suivant [3]:

- Dans une opération d'absorption gaz-liquide, une certaine quantité de gaz $A$ est transférée dans la phase liquide à travers l'interface.

-- Selon l'absence ou la présence de corps chimique réactif $B$, le gaz dissous dans la phase liquide ne 
réagit pas (cas de l'absorption physique simple) ou réagit chimiquement.

-- En fait, en choisissant de façon judicieuse les quantités et la composition du réactif ainsi qu'en contrôlant les conditions d'expériences (température, $\mathrm{pH} .$. ) on se trouve en présence à la fois d'un transfert de matière et d'une réaction chimique.

-. Selon que l'un ou l'autre de ces deux phénomènes est le plus lent, on pourra déterminer respectivement les coefficients de transfert de matière ou l'aire interfaciale.

Analytiquement, on établit les variations du "facteur d'accélération" $E$ (équivalent au rapport : flux d'absorption chimique/flux d'absorption physique) en fonction du nombre de Hatta, $H a$ (équivalent au rapport : flux d'absorption en régime diffusionnel et réactionnel/flux d'absorption en régime diffusionnel seulement) ainsi défini :

$$
H a=\frac{\sqrt{D_{A} K C_{B}}}{K_{L}}
$$

où $D_{A}$ est le coefficient de diffusion du gaz $A$ dissous dans le liquide, $K$ la constante de vitesse de la réaction et $C_{B}$ la concentration du réactif $B$ au sein du liquide. Ces variations sont donc représentatives de la compétition qui se forme entre le transfert de gaz sans réaction chimique - cas du régime diffusionnel - et la réaction chimique elle-même. En d'autres termes, si on favorise le régime diffusionnel on peut déterminer les coefficients de transfert globaux du soluté, par contre si on privilègie la réaction chimique on peut déterminer l'aire interfaciale.

\section{Méthode électrochimique : mesure du frotte- ment pariétal en écoulement diphasique}

Cette méthode a été mise au point et testée au laboratoire d'Energétique et de Mécanique Théorique et Appliquée de l'Institut National Polytechnique de Lorraine (à Nancy) par Messieurs G. Cognet, M. Lebouché et M. Souhar (Voir référence [4]).

La détermination du frottement pariétal est une étape fondamentale dans l'établissement des bilans de quantités de mouvement et cette détermination est plus délicate en écoulement diphasique qu'en écoulement monophasique. La méthode électrochimique permet de calculer ce frottement dans le cas d'écoulements gazliquide. C'est une mesure locale et instantanée.

Son principe peut être simplement défini par analogie avec une méthode maintenant bien connue et exploitée : l'anémothermométrie à fil ou film chaud. C'est en effet l'équivalent en transfert de masse du transfert de chaleur utilisé en anémométrie. Au lieu du refroidissement (fonction de la vitesse de l'écoulement) d'un élément sensible chauffé, on utilise l'électrolyse rapide d'un réactif sur une microélectrode affleurant la paroi inerte intérieure du conduit où s'effectue l'écoulement du fluide.
Le courant limite d'électrolyse $I$, qui est proportionnel au flux de réactif à l'électrode, est une fonction du gradient de vitesse local à la paroi $(\partial U / \partial y)_{y=0}$ (avec $U:$ vitesse parallèle à la paroi et $y$ : direction perpendiculaire à la paroi).

On sait que cette valeur du gradient à la paroi est reliée simplement à la contrainte de frottement pariétal par : $\tau=\mu(\partial U / \partial y)_{y=0}$ où $\tau$ est la contrainte de frottement et $\mu$ la viscosité dynamique du fluide. L'intensité $\mathrm{du}$ courant varie selon la puissance $1 / 3$ de la valeur du gradient de vitesse à la paroi (voir références [4] et [5]). L'utilisation de deux électrodes identiques placées l'une en aval de l'autre (espacement de $2 \mu$ ) dans la direction de l'écoulement permet de déterminer le sens de cet écoulement à chaque instant. De mème que la présence d'un premier fil chaud peut affecter le fonctionnement d'un second fil placé dans son sillage thermique, la seconde électrode sera affectée dans son fonctionnement par le sillage massique de la première (Voir fig. 1).
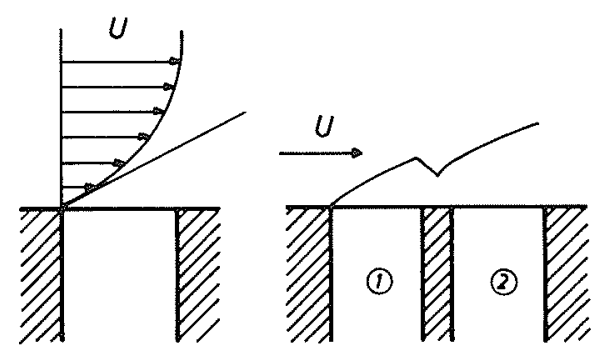

$$
\begin{aligned}
& \text { si } U_{12} \rightarrow I_{1}-I_{2}>0 \\
& \text { si } U_{21} \rightarrow I_{1}-I_{2}<0
\end{aligned}
$$

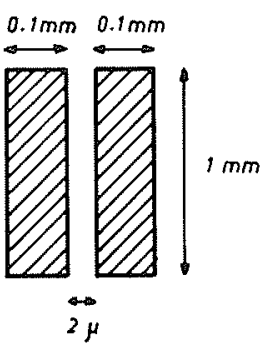

Figure 1. - Méthode électrochimique : Principe de fonctionnement et dimensions des électrodes.

La méthode décrite ci-dessus dans le cas des écoulements de fluide monophasiques liquides a été appliquée sans difficultés à des écoulements diphasiques gaz-liquide à bulles pour lesquels la paroi reste baignée en permanence par le liquide.

La présence de bulles d'air ou de bouchons va naturellement affecter le frottement pariétal dont les fluctuations seront enregistrées par les électrodes. L'utilisation de sondes avec électrodes doubles permettra même de remarquer les inversions de courant dans le régime d'écoulement à bouchons.

Les résultats tests de cette méthode ont été concluants [5]. 
Sondes optiques : détermination de la fonction indicatrice de phase en écoulement diphasique finement dispersé

Cette technique a été mise au point dans les laboratoires de la Société "Assistance Industrielle Dauphinoise" à Grenoble par Monsieur F. Danel.

Il s'agit d'une extension de techniques déjà développées par le même auteur en collaboration avec Monsieur J.M. Delhaye (C.E.A.) [6]. Le principe de base est simple : il consiste à mesurer la lumière refléchie par l'extrémité taillée en pointe de la sonde. Selon les indices de réfraction relatifs de la partie sensible de la sonde et de la phase en contact, le faisceau incident est soit réfracté, soit totalement réfléchi (voir fig. 2 a). Il s'agit donc d'une méthode "tout ou rien" particulièrement efficace pour la détection de phase ; c'est une mesure locale de la fonction indicatrice de phase.

Comme pour toutes les sondes locales matérielles, on doit considérer le problème de la perturbation apportée à l'écoulement par la présence de la sonde, problème d'autant plus crucial en écoulement diphasique que la phase dispersée est plus fine. Les bonnes performances obtenues par l'anémothermomètre à film chaud [7] sont limitées, en raison des dimensions de ce type de sonde, à des diamètres de bulles relativement importants $(>0.5 \mathrm{~mm})$.

Le problème de la miniaturisation prend alors toute son acuité pour la mesure en écoulements finement dispersés (émulsions, aérosols...). C'est pour répondre à

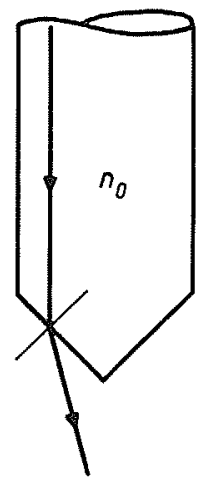

Liquide $n_{L}>1.15$

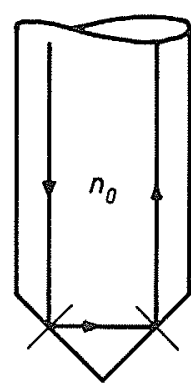

(a)

$$
\text { Gaz } \quad n_{G}<1.15
$$

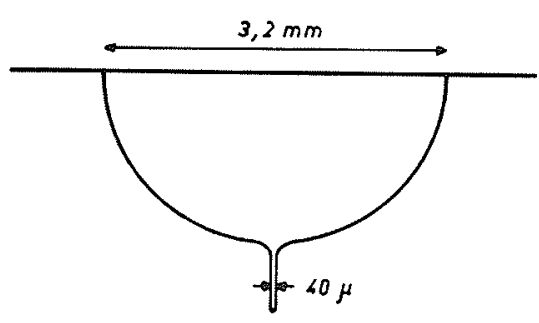

(b)

Figure 2. - a) Principe de fonctionnement des sondes optiques. b) Interaction goutte-sonde. ce souci que des sondes optiques monofibres ont été conçues. Leur diamètre est de $40 \mu$, avec un diamètre de la partie sensible réduit à $20 \mu$ (à comparer aux sondes à fibres repliées en $U$ de la génération précédente de $100 \mu$ de diamètre). La lumière incidente provient d'un faisceau laser (de faible puissance $<1 \mathrm{~mW}$ ) focalisé sur une section droite de la monofibre. Après un trajet aller-retour dans la fibre unique, le signal retour est extrait, puis focalisé sur un phototransistor muni d'un amplificateur. Le temps de réponse est très faible, de l'ordre de quelques dixièmes de microsecondes ce qui permet une détection assez nette de l'arrivée ou du départ de la bulle ou goutte sur la sonde (voir fig. $2 b$ ).

La forme géométrique de l'extrémité sensible de la sonde a été l'objet d'études poussées.

Des formes en ogive rendent la sonde sensible à la variation de l'indice optique du liquide lorsque l'on se trouve en phase liquide. On pourra donc effectuer des détections liquide-liquide avec ce modèle. Des formes coniques ne permettent pas une sensibilité aussi élevée mais sont très bien adaptées à la détection gaz-liquide, (sondes fonctionnant en tout ou rien).

La caractérisation complète de telles sondes est en bonne voie à l'heure actuelle.

Méthode optique de diffusion à 90 degrés : mesures sur les écoulements finement dispersés

Cette méthode a été conçue et mise au point à l'Institut de Mécanique Statistique de la Turbulence de l'Université d'Aix-Marseille II, par Messieurs F. Avellan, et $F$. Resch [8, 9]. Le prototype a été réalisé par la société A.I.D.

Une particule est éclairée par un faisceau laser dans des conditions connues ; le flux diffusé par cette particule dans une direction perpendiculaire au rayon incident est uniquement fonction du diamètre de la particule. Il suffit donc d'étalonner la sonde dans une gamme suffisante de diamètres pour pouvoir déterminer la granulométrie de la suspension. On peut ainsi détecter des particules dont la taille varic entrc quelques microns ct quelques millimètres. C'est une sonde locale. L'utilisation d'optiques à immersion universelle permet de l'employer indifféremment en phase gazeuse pour la mesure des gouttelettes liquides (par exemple aérosols) ou en phase liquide pour détecter de petites bulles d'air.

Le schéma de principe est représenté sur la figure 3. La source lumineuse est composée d'un tube laser $\mathrm{He}-\mathrm{Ne}$ de faible puissance $(2 \mathrm{~mW})$. Le flux diffusé est mesuré par un photorécepteur.

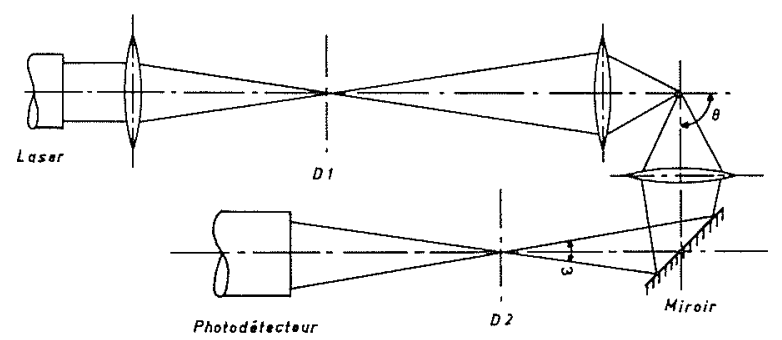

Figure 3. - Méthode optique de diffusion : Schéma de principe. 
Les premiers tests sur des aérosols monodispersés calibrés ont été positifs. Le développement actuel concerne la détermination exacte du volume de mesure et le calcul des "effets de bord". Une installation d'étalonnage a été construite et permettra très prochainement d'étalonner le prototype de la sonde. Les auteurs veulent, par cette méthode, mesurer les granulométries d'aérosols marins produits par l'éclatement de bulles d'air à l'interface air-mer ; ces bulles d'air étant ellesmêmes produites par le déferlement des vagues.

Traitement numérique du signal de sortie d'un anémomètre à film chaud en écoulement diphasique.

Cette étude a été effectuée dans le cadre d'une coopération franco-canadienne (Université de Toronto, Ontario, Canada) par Messieurs R. Abel et F. Resch [10].

Le traitement numérique d'un signal d'un anémothermomètre à film chaud, placé dans un écoulement diphasique gaz-liquide, a déjà été effectué par $F$. Resch dans le cas d'un écoulement à bulles [7] et [11]. Les auteurs ont voulu l'étendre au cas des écoulements à poches et le généraliser au cas mixte bulles-poches.

Dans le cas d'un écoulement à poches le signal de sortie est représenté schématiquement sur la figure 4 . Il est à remarquer que les différences de tension $\Delta V_{1}$ et $\Delta V_{2}$ sont des fonctions croissantes du temps de passage des poches sur la sonde : $\Delta V=f(t)$. Leurs variations sont représentées sur la figure 5 dans le cas d'un écoulement avec poche de vapeur en conduite horizontale. $\Delta V_{1}$ correspond à la chute de tension due à l'arrivée de la première interface de la poche gazeuse sur l'élément sensible (début de poche) ; $\Delta V_{2}$ correspond à la brusque remontée due au passage de la seconde interface lorsque la sonde se trouve de nouveau en phase liquide (fin de poche).

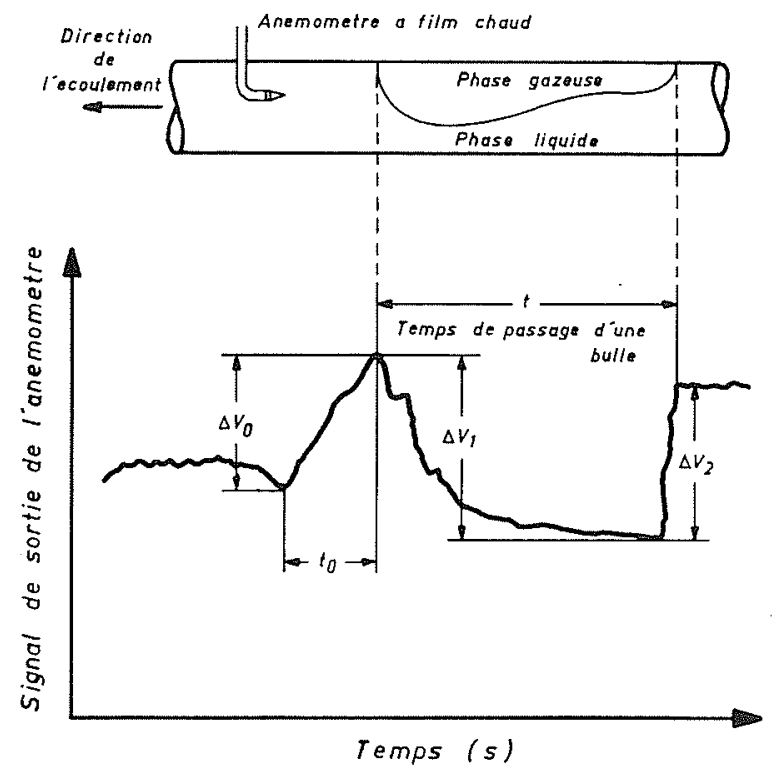

Figure 4. - Représentation schếmatique du signal de sortie d'un anémothermomètre à film chaud dans le cas d'un écoulement à poches.

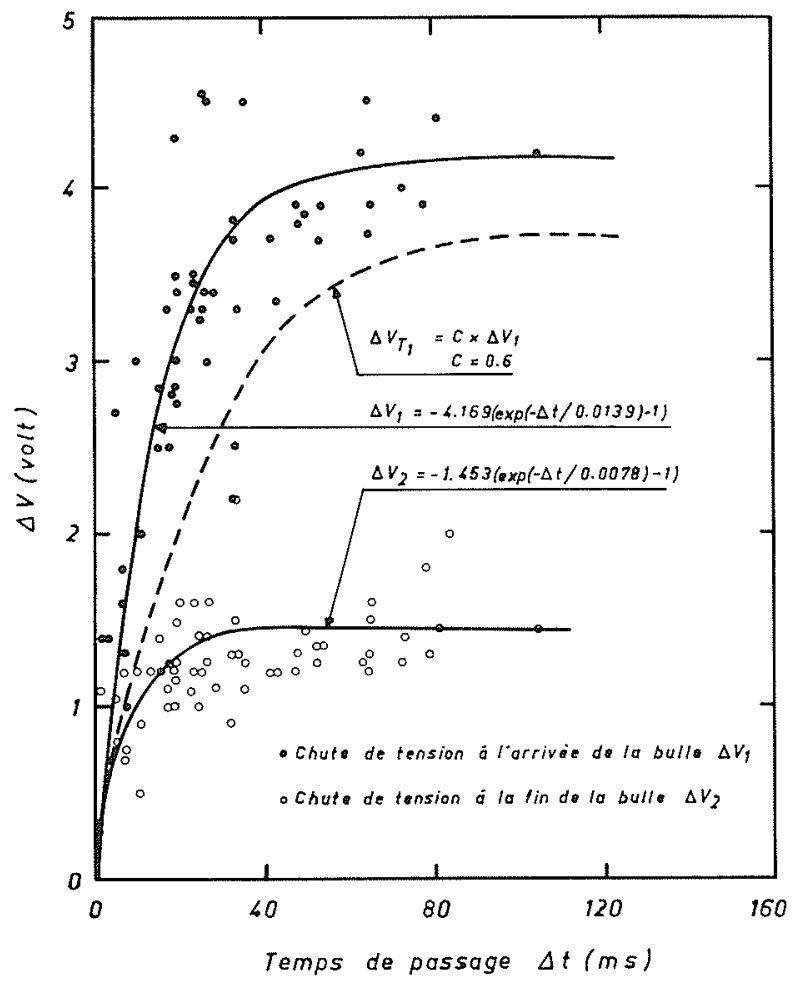

Figure 5. - Variations des différences de tension avec la durée de passage des poches.

Les différentes valeurs de $\Delta V_{1}$ et $\Delta V_{2}$ sont comparées à un seuil $\Delta V_{s}$ tel que $\Delta V_{s}=C \Delta \stackrel{V}{V}$ avec $C$ un coefficient d'atténuation. Ceci permet une discrimination entre les parties du signal correspondant aux fluctuations turbulentes du liquide et celles correspondant à la phase gazeuse. La difficulté réside dans le choix de ce facteur d'atténuation. Abel et Resch ont proposé une méthode qui consiste à établir "à priori" la courbe de variation de ce coefficient $C$ en fonction du taux de présence de la phase gazeuse (taux de vide), voir figure 5 . $\mathrm{Si}$ ce coefficient est trop fort, toutes les variations de tension seront attribuées à des fluctuations turbulentes

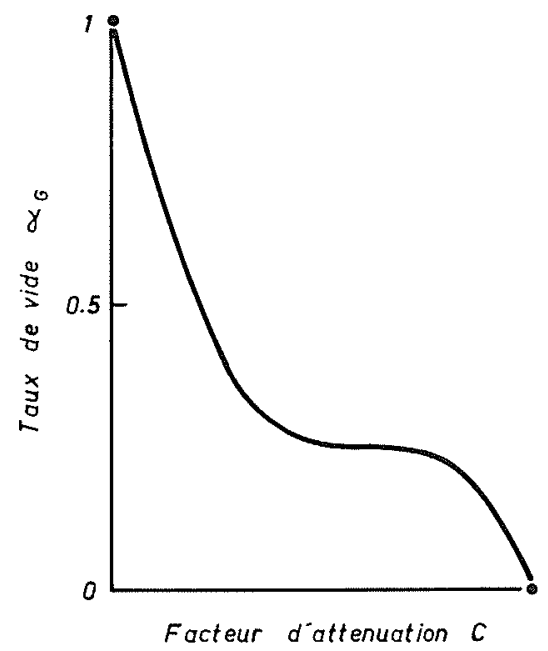

Figure 6. - Variation du taux de vide mesuré en fonction du facteur d'atténuation $C$. 
de la vitesse du liquide $\left(\alpha_{G}=0\right)$. Si ce coefficient est trop faible, toutes les variations seront assimilées au passage de poche de gaz $\left(\alpha_{G}=1\right)$. Entre ces deux valeurs extrêmes, on observe une variation continue et la présence d'un "plateau" horizontal permettra d'attribuer une valeur correcte au coefficient de discrimination $C$ (voir fig. 6).

Cette méthode a été ensuite étendue au cas des écoulements à bulles et au cas des écoulements mixtes bullespoches. Comme pour toute méthode locale, rappelons qu'une méthode globale est nécessaire pour en confirmer la validité ou "caler" les seuils de détection.

\section{Conclusion}

Bien que choisies de façon subjective, comme indiqué au début de cet exposé, les méthodologies proposées offrent un bon aperçu des récents développements concernant la métrologie des écoulements diphasiques. Il faut espérer que les années à venir permettront de classer ces méthodes comme "anciennes" et de voir apparaitre des développements encore plus audacieux et plus précis.

\section{Remerciements}

Les études sur les écoulements diphasiques entrent dans le cadre de recherches effectuées à l'IMST et soutenues par le Centre National de la Recherche Scientifique et le Centre National pour l'exploitation des Océans (contrat CNEXO/IMST $n^{\circ} 1869$ ). Nous remercions vivement ces deux organismes pour leur aide morale et financière.

\section{Références}

[1] Métrologie des Ecoulements diphasiques. - La Houille Blanche $\mathrm{n}^{\circ} 5 / 1978$ - Séminaire "Métrologie des écoulements diphasiques" (animateur : F. RESCH), Groupe de Travail "Ecoulements polyphasiques" de la S.H.F

[2] LAURENT A., PROST C. et CHARPENTIER J.C. - Détermination par méthode chimique des aires interfaciales et des coefficients de transfert de matière dans les divers types d'absorbants et de réacteurs gaz-liquide, Journal de Chimie Physique, 1975, $\mathrm{n}^{\circ} 2$, pp. 236-244.

[3] DANCKWERTS P.V. - Gas-liquid reactions, Ed. McGrawHill Book Company, London, 1970.

[4] COGNET G., LEBOUCHE M. et SOUHAR M. - Utilisation des techniques électrochimiques pour la mesure du frottement pariétal dans les écoulements diphasiques, La Houille Blanche $\mathrm{n}^{\circ} 5 / 1978$.

[5] SOUHAR M. et COGNET G. -- Electrochemical Method for Dynamic Measurements in Two-Phase Flow, Dynamic Flow Conference, Marseille, Sept. 1978. A paraître dans les proceedings.

[6] DANEL F. et DELHAYE J.M. - Sonde optique pour la mesure du taux de présence local en écoulement diphasique, Mesure, Régulation, Automatisme; Août-Septembre 1971, pp. 99-101.

[7] RESCH F.J., LEUTHEUSSER H.J. and ALEMU S. Bubbly Two-Phase Flow in Hydraulic Jump. Journal of Hydraulics Division, ASCE, vol. 100, $\mathrm{n}^{\circ} \mathrm{HY} 1$, Proc. Paper 10297 , pp. 137-149.

[8] AVELLAN F. - Méthodes de mesure des caractéristiques diphasiques au voisinage de l'interface air-mer, Rapport d'activités D.E.A. I.M.S.T. Université d'Aix-Marseille 1978

[9] RESCH F. - Measurement Techniques in the Two-Phase Flow Region of the Air-Sea Interface Layer : Dynamic Flow Conference, Marseille septembre 1978. A paraitre dans les proceedings.

[10] ABEL R. and RESCH F.J. - A method for the Analysis of Hot-Film Anemometer Signals in Two-Phase Flows, Intern. Journ. of Multiphase Flow, vol. 4, $\mathrm{n}^{\circ}$ 5/6, Dec. 1978, pp. 523-533.

[11] RESCH F.J. and LEUTHEUSSER H.J. - Le ressauthydraulique : mesures de turbulence dans la région diphasique, $L a$ Houille Blanche $\mathrm{n}^{\circ} 4,1972$, pp. 279-293. 


\section{Discussion}

Président : M. le Professeur FORTIER

M. Le Président. - Je remercie M. RESCH de son excellente communication. Est-ce qu'il y a des questions à poser ?

M. FITREMANN. - Je suis personnellement intéressé par la détection des poches et l'anémométrie utilisée pour cette détection. Dans votre expérience, quelles étaient la dimension de l'installation et la taille des poches observées?

M. RESCH. - La méthode de séparation a été mise au point en commun avec R. ABEL. Il l'a exploitée dans sa thèse. Il s'agissait d'un écoulement en conduite de section circulaire de 30 mètres de long et $75 \mathrm{~mm}$ de diamètre. La longueur des poches de vapeur était de l'ordre de 10 à 20 centimètres.

J'avais déjà proposé une méthode de séparation des phases pour le cas d'un écoulement à bulles; R. ABEL voulait effectuer une séparation des phases dans le cas d'un écoulement à poches. Nous avons donc essayé de généraliser la première méthode. Nous avons maintenant les moyens numériques d'effectuer cette séparation pour les deux classes d'écoulement et même en écoulement mixte bulles-poches. C'est là que réside l'intérêt de la méthode.

M. $B A B Y$. - Pensez-vous que la méthode optique de diffusion est adaptable à la mesure de la taille et du nombre des gouttes qui peuvent être entraînées dans une tour de réfrigération humide ? Ceci serait utile pour déterminer l'efficacité du système de séchage.

M. RESCH. - Cette sonde optique a été conçue pour détecter des particules dans une gamme de quelques microns à quelques millimètres. Par ailleurs, je n'ai pas fait remarquer dans mon exposé que l'emploi d'optiques à immersion universelle permettait à cette sonde d'être utilisée aussi bien en phase liquide pour la détection des bulles de faible diamètre qu'en phase gazeuse pour la détection des petites gouttelettes d'eau (comme les aérosols).

En ce qui concerne les nuages il faudrait pouvoir détecter des diamètres encore plus fins.

M. $B A B Y$. - Quel est le poids de l'appareil ?

M. RESCH. - De l'ordre de deux kilogrammes.

M. DELHAYE. - L'étude de la séparation des gouttelettes d'un écoulement dispersé nécessite souvent la mesure de diamètres de gouttelettes supérieurs au millimètre. Dans ce cas les méthodes basées sur la diffusion ne semblent pas devoir donner satisfaction. En revanche la granulométrie et la vitesse de ce type de gouttelettes peuvent être déterminées à l'aide,

- soit d'un faisceau laser découpé en lamelles par une grille de

Ronchi et un photodétecteur (DUKLER A.E., 1978, Twophase interactions in countercurrent flow, Annual report for US. NRC)

- soit de l'ombroscopie laser (méthode mise au point à Harwell, Grande-Bretagne, par G.F. HEWITT et ses collaborateurs)

$M$. le Président. - Est-ce qu'il faut un laser et un détecteur?...

M. DELHAYE. - Le principe est le même que celui de l'anémométrie laser, mais on découpe un seul rayon avec une grille et les particules passant dans la grille sont éclairées plus ou moins vite suivant la vitesse. Nous avons des problèmes avec les gros diamètres.

M. RESCH. - Tout dépend de la taille du volume de mesure. Remarquons seulement que pour des bulles ou gouttes de 4 à $5 \mathrm{~mm}$ de diamètre on dispose de l'anémométrie à film chaud qui est une méthode assez précieuse et qui donne de bons résultats.

M. LECOFFRE. - Je voudrais faire deux remarques au sujet de la méthode par diffraction, technique utilisée depuis quelques années chez NEYRTEC.

D'une part, les problèmes ne sont pas les mêmes en ce qui concerne les gros et les petits diamètres de bulles. Pour les petits diamètres, c'est un problème d'intensité lumineuse qui se pose ; pour les gros diamètres, la difficulté est liée à la précision de définition du volume de mesure.

D'autre part, on peut améliorer la précision et la sensibilité en utilisant une optique qui soit une optique de restitution très ouverte à $90^{\circ}$, ou qui soit une optique de restitution qui ne soit plus à $90^{\circ}$, mais aux alentours de 12 ou $18^{\circ}$ par rapport à l'axe đ'éclairage.

M. RESCH. - Je suis d'accord avec vous, on peut très bien utiliser la diffusion sous un angle différent.

M. DELHAYE. - Pour la détermination des gouttelettes on pourrait aussi utiliser l'ombroscopie laser mise au point à Harwell par Hewitt et ses collaborateurs.

J'ai vu un film sur l'entraînement de gouttelettes à partir d'un film ruisselant par un contre-courant de gaz, où l'on peut mesurer de façon très précise les diamètres des gouttelettes compte tenu d'une correction due aux effets d'ombre.

M. RESCH. - Par atténuation du faisceau ?

M. DELHA YE. - La géométrie était un tube, avec un film ruisselant sur les parois intérieures du tube.

On envoie un courant de gaz à contre-courant ; on a un éclairage axial par un faisceau laser élargi qui fait tout le diamètre du tube, et on récupère ce faisceau à la sortie du tube, l'astuce consistant à dévier le courant de gaz et le courant de gouttelettes à la sortie du tube.

M. MONDIN. - Je voudrais faire une intervention sur le raccordement en quelque sorte entre ces mesures locales et la théorie, et les paramètres qui interviennent dans les équations.

Historiquement, la meilleure mesure locale qu'on ait pu faire, c'est l'indication de phase par des sondes optiques ou des sondes d'impédance. En combinant deux sondes l'une à côté đe l'autre on a pu mesurer le temps de parcours d'une interface entre ces deux sondes et avoir ce qu'on appelle la vitesse de transit d'interface.

Lorsqu'on a affaire à un écoulement de petites bulles de gaz dans de l'eau il est évident que cela peut servir à démontrer que la vitesse à l'interface est égale à la vitesse de la phase gazeuse. Lorsqu'on a affaire à un écoulement de petites gouttes dans un gaz, cette vitesse d'interface est la vitesse du liquide.

Mais entre les deux on ne sait pas utiliser la vitesse qu'on mesure. En particulier on ne sait pas faire figurer dans les équations cette vitesse de transit d'interface.

Or il se trouve que depuis ces dernières années les techniques ont progressé, et désormais on sait mesurer la vitesse locale instantanée dans la phase liquide. On ne sait pas si l'on est capable de la mesurer dans tous les cas, mais enfin on est proche du moment où l'on dominera parfaitement cette mesure.

Actuellement il y a un problème mathématique, un problème de cinématique aléatoire extrêmement intéressant, que je pose à des fondamentalistes mathématiciens et qui est le suivant :

On connait à chaque instant la vitesse de transit à l'interface et la vitesse locale de la phase liquide ; comment en déduire la vitesse locale instantanée de la phase gazeuse?

Si l'on arrive à résoudre ce problème qui est sûrement très difficile, on fera faire un progrès considérable au raccordement entre l'expérimentation et la théorie.

$M$. RESCH. De même que l'on peut utiliser un anémomètre à film chaud pour déterminer les vitesses instantanées de la phase liquide, on peut utiliser cette même technique en inversant les phases et en utilisant un fil chaud.

M. MONDIN. Est-ce que vous pensez que cela pourrait fonctionner avec un système à petites bulles?

M. RESCH. Je reconnais que l'utilisation d'une sonde matérielle aux dimensions imposées a toujours des limites et que seule la miniaturisation poussée de telles sondes permettra de détecter des petites bulles. 


\section{Abstract}

\section{Recent progress in two-phase metrology}

This review of recent progress in two-phase metrology is by no means exhaustive, having deliberately been limited to the description of a few methods and techniques developed in France during the last few years. Some of these have already been presented at Author's Seminary on Two-Phase Metrology (see special issue of $\mathrm{La}$ Houille Blanche No. 5, 1978).

In this report, recent developments are discussed under the following headings :

1. Measurement of interface areas and material transfer coefficients by a chemical method

This method was developed and first applied by Charpentier, Laurent and Prost at the 'Ecole Nationale des Industries Chimiques' in Nancy. It is a global method for determining interface area and material transfer coefficients. A gasliquid absorption process can take place without a chemical reaction (diffusion) or with one. Transfer coefficients can be determined from the former process, interface area from one with reaction.

II. Measurement of two-phase flow boundary friction by an electro-chemical method

This method was designed and tested by Cognet, Lebouche and Souhat at the 'Laboratoire d'Energétique et de Mécanique Théorique et Appliquée' in Nancy. It is an instantaneous, local measurement method based on the principle of the mass transfer equivalent of heat transfer applied in anemometry. The limit of electrolytic current produced by contact of two electrodes embedded in a pipe wall is a simple function of the local velocity gradient at the boundary. By using two electrodes, the direction of flow can be determined.
III. Determination of the phase-indicating function in finely dispersed two phase flow by an optical refraction method

This method was developed by Danel in the 'Assistance Industrielle Dauphinoise' Company's laboratory. It is an extension of an earlier method designed by Danel and Delahaye (C.E.N.G.), using more efficient, miniaturized $40 \mu$ diameter probes $(20 \mu$ at the sensitive tip) with a $1 / 10$ th microsecond response.

IV. Determination of air-bubble or water droplet size by an optical diffusion method

This method was designed and developed by Avellan and Resch at the 'Institut de Mécanique Statistique de la Turbulence' of Aix-Marseilles University. The prototype was produced by the A.I.D. Company.

This is a local measurement method of the dimensions of bubbles or droplets passing through the measurement space. The flux diffused by each bubble or droplet in a perpendicular direction to the incident ray depends solely on the bubble or droplet diameter. The particle diameter detection range is from a few microns up to approximately a millimetre.

$V$. Numerical processing of a hot-film anemometer output signal in cavity flow

This study was carried out by Abel and Resch at Toronto University under a joint French-Canadian cooperation scheme. The method is a numerical method whereby gas and liquid phases in cavity flow (or mixed flow with bubbles and cavities) can be identified by processing the output signal from a hotfilm anemometer in the flow. 